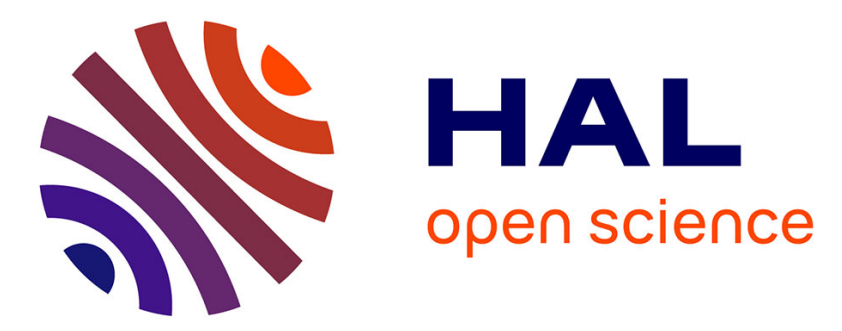

\title{
La magistrature à l'épreuve de la guerre d'indépendance algérienne
}

\author{
Sylvie Thénault
}

\section{To cite this version:}

Sylvie Thénault. La magistrature à l'épreuve de la guerre d'indépendance algérienne. Outre-Mers Revue d'Histoire, 2003, 90 (338), pp.153-162. 10.3406/outre.2003.4020 . hal-02355858

\section{HAL Id: hal-02355858 \\ https://hal.science/hal-02355858}

Submitted on 8 Nov 2019

HAL is a multi-disciplinary open access archive for the deposit and dissemination of scientific research documents, whether they are published or not. The documents may come from teaching and research institutions in France or abroad, or from public or private research centers.
L'archive ouverte pluridisciplinaire HAL, est destinée au dépôt et à la diffusion de documents scientifiques de niveau recherche, publiés ou non, émanant des établissements d'enseignement et de recherche français ou étrangers, des laboratoires publics ou privés.

\section{(이) $\$$}

Distributed under a Creative Commons Attribution - NonCommercial - NoDerivatives| 4.0 


\title{
La magistrature à l'épreuve de la guerre d'indépendance algérienne
} Sylvie Thénault

\section{Résumé}

Les modalités du service de l'État par les magistrats en poste en Algérie pendant la guerre d'indépendance ont pris deux aspects : I'application de la législation et l'exercice de fonctions nouvelles. L'intense activité répressive des tribunaux et l'absence de condamnations de policiers ou militaires coupables de torture prouvent la participation du personnel de la Justice à cette guerre. Il faut dire qu'à l'époque, il n'existait ni formation professionnelle ni syndicat susceptible de les orienter vers la critique de leurs pratiques et la prise de distance avec le pouvoir politique. Ces données ont joué comme facteur aggravant alors que la majorité de ces magistrats étaient eux-mêmes natifs du sol algérien.

\begin{abstract}
During the Algerian War, the magistrates were involved in the repression of the nationalists and their supporters : they had to enforce the law decided in France and they were recalled by the army. Hundreds of militants, terrorists, fighters... were introduced into courts but no French soldier or policeman had been charged of torture. This proves that the magistrates tookpart to this War. Since they didn't receive any professionnal formation and didn't have any trade-union, they couldnt be independant from the policital power. And the majority of them were born in Algeria, which they considered as their own country.
\end{abstract}

Citer ce document / Cite this document :

Thénault Sylvie. La magistrature à l'épreuve de la guerre d'indépendance algérienne. In: Outre-mers, tome 90, n³38-339, 1er semestre 2003. l'Etat et les pratiques administratives en situation coloniale. pp. 153-162;

doi : https://doi.org/10.3406/outre.2003.4020

https://www.persee.fr/doc/outre_1631-0438_2003_num_90_338_4020

Fichier pdf généré le 26/04/2018 


\title{
La magistrature à l'épreuve de la guerre d'indépendance algérienne
}

\author{
Sisir THE.VIIT
}

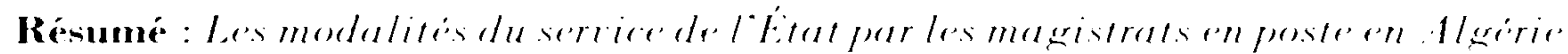

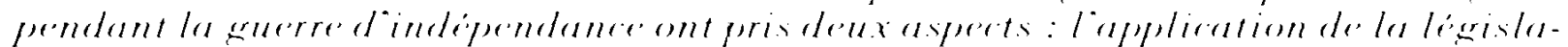

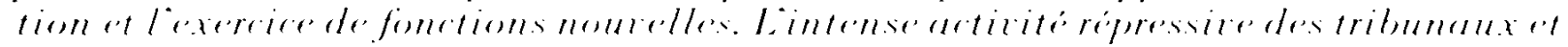

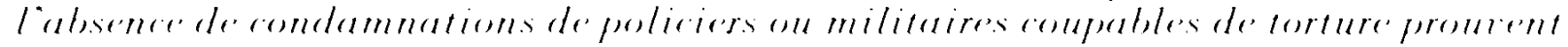

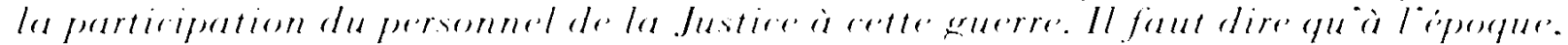

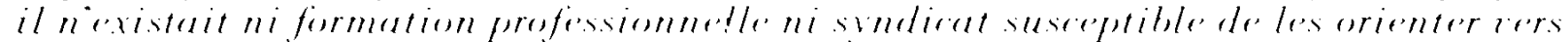

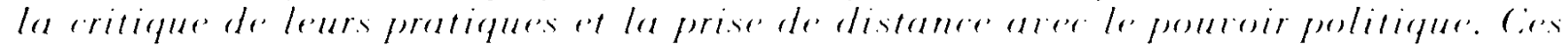

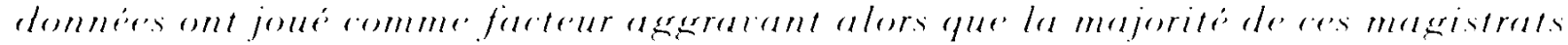
itaient aux-mimes matifis du sol algérien.
\end{abstract}

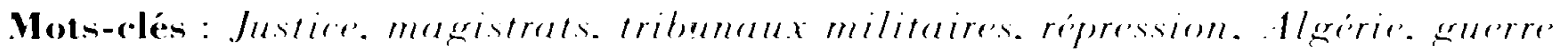
d'Mlérie.

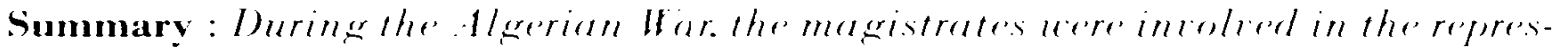
sion of the nationalists and the ir supporters : the v had te enforre the late derided in

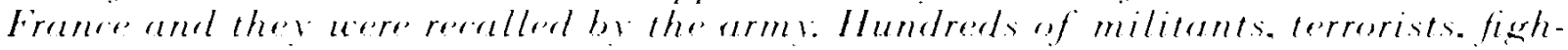

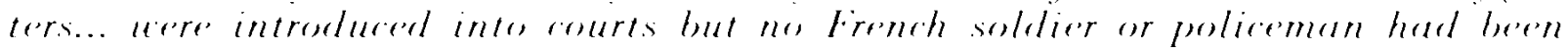

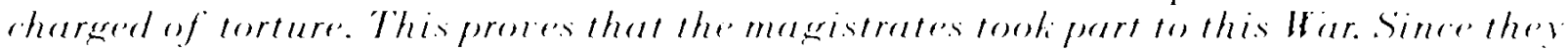

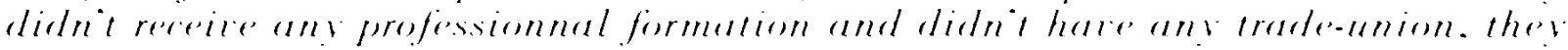

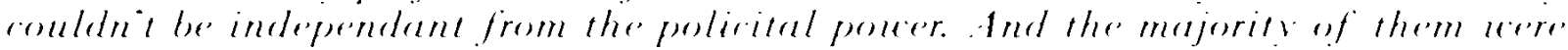

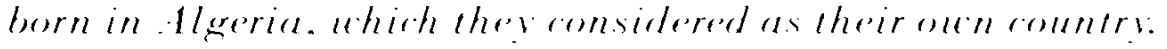

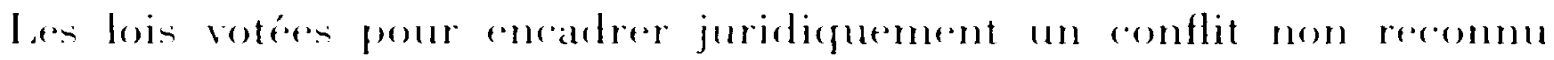
comme une guerreont mis les magist rats a l'epreure en les faisant participer a la lutte contre lennemi. consideré eomme un simple délinquant on reviminel. Ces lois ont aménagé en Algérie. 111 systeme de répersion melant la justice rivile et la justice militaire. dont la eompetence a sans resse éte ilargite.

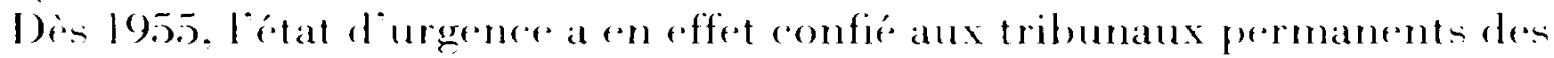
forces armése (TPPA) le jugement de tous les " crimes" commis par les nationalistes algériens. I a justice ejvile conservait rependant le déelenchement des poursuites et l'instruction des affaires tandis que les tribunaus

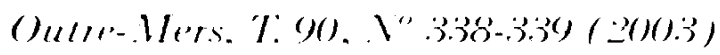


correctionnels jugeaient les aretes qualifiés de delits. Fin 1956. les décrets adeptés grâce aux pouroirs spéciaux ont reconduit ces mesuresen les aggravant. puisque les TPFA ont été autorisés a revendiquer l'instruction des affaires. Par ailleurs, une nouvelle procedure expéditive, fut instaurée : la "traduction directe". Flle permettait de traduire devant les TPFA, sans instruction préalable, tout individu pris en flagrant délit. Finfin, avec le décret du 12 févier 1960 creant la fonction de procureur militaire, la procédure a eté totalement remodelée. Ces procureurs militaires etaient des magistrats rivils rappeles sous les drapeaux et rhargés de mener une enguête sur les persommes arretées par l'armée. Suls s'aréraient eoupables, ils étaient trarluits devant les TPFA. Instruction supprimée, tribunaux correctionnels écartés: la justice civile nest plus intervenue jusqu'a la fin du conflit.

Appliquer et interpréter la loi somt les deux tâdes traditionmellement dévolues au personnel de la justice. Elles sonserivent dans un rapport classique et ordinaire entre le pousoir legislatif le pouvoir exécut if et le pouvoir judiciaire. rhareun jouant son role. Lri, les réactions des magristrats à lapplication d'une procédure étendant la compétence des TPFA constituent lesecentiel de leur rapport aver les textes votés pour l' Algerie pendant la guerre. Restreindre lanalyse a cet aspect ne suffit eependant pas : comme le prouve le décret du 12 févier 1960 , les magist ratsont aussiété mobilisés au premier sens du terme, puisquils ont été rappelés sous les drapealux. Outre cette fonction de procureur militaire, ils ont aussi assumé la présidence des TPFA, en étant rappeles sous les drapeaux, ou en sus de leurs fonctions habituelles, sans etre rappeles selon les periodes de la guerre. Pour un magistrat en poste en Algerie entre 1951 et 1962. ou envové de lautre coté de la Méditerranée pour les besoins de la guerre. le serviere de l'Etat a ainsi pris une double signification : appliquer et interpréter les textes, certes. mais aussi exercer des fonctions nouvelles.

Comment les magistratsont-ils appréhendé cette participation à leffort de guerre contre les nationalistes algériens? Fn quoi leur recrutement local a-t-il pu constituer un élement discriminant du eomportement des magistrats? Au contraire des autres colonies frangaises, en effet, les magistrats exergant en Algérie ne formaient pas un corps spécifique : le recrutement, la formation, les reriteres de mutation. nomination, promotion... étaient identiques et ne les séparaient pas de la magistrature métropolitaine. En thérie. tous les mourements étaient possibles doun territoire a l'autre : recruté en metropole, un magistrat pouvait etre affecte en Algérie et inversement.

\section{Appliquer et interpréter les textes}

I es arehives du ministere de la Justice permettent de savoir eomment le transfert de compétence vers la justiere militaire a ete mis en praticfue par les procureurs généraux d' Xlger. d'Oran et de Constantine. Fin l'areceptant sans difficulte, ils ont optimise son applieation. C'est ainsi qu'a Alger, Jean Reliquet a fait pression sur les autorités parisiennes pour obtenir le renforeement 


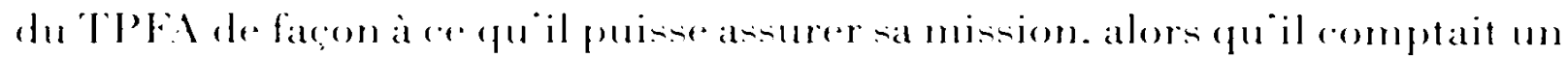
personmel insuffisant. Jean Reliquet lui a meme prete des locaux et détaché

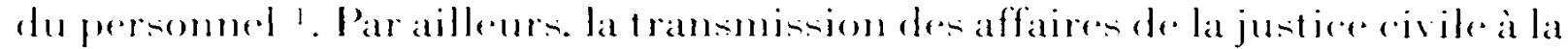

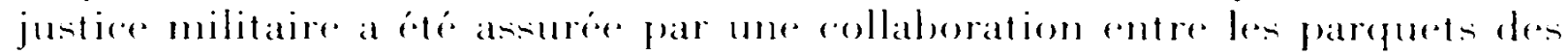

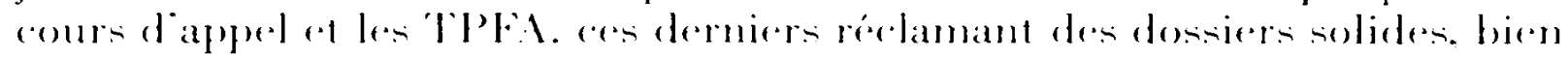
eomstruits pour promoneer des jugements exemplaires. Au parquet général

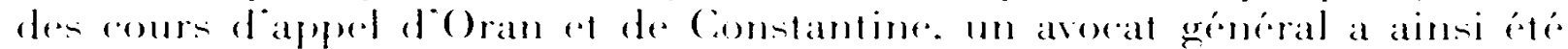

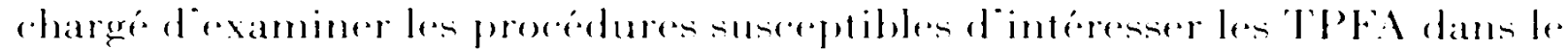

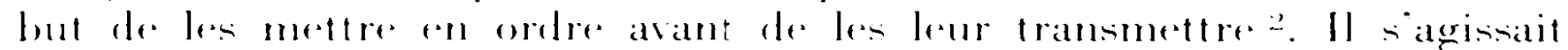

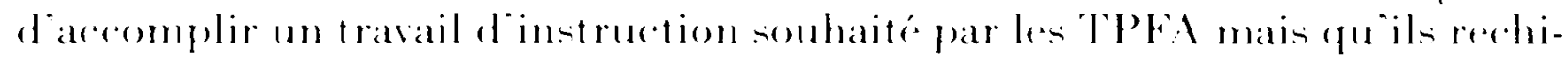

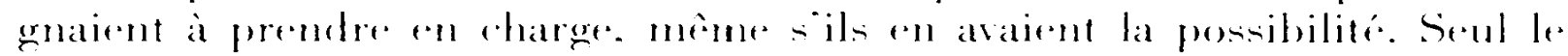

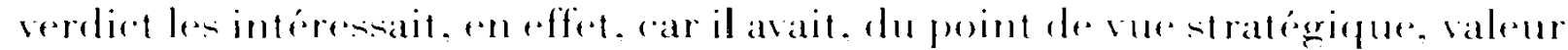
dexemple : il état suseptible d"influenerer la population au eontraire de

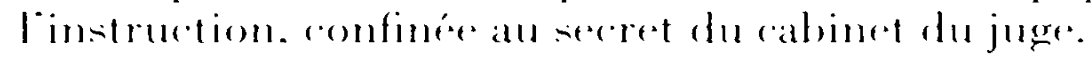

Interrogés a posteriori sur leur sentiment fare a cette intervention de la justice militaire. deanciens juges d instruetion dementent avoir ressenti une.

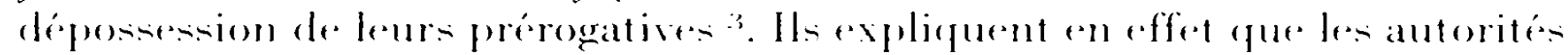
militaires nontervenaient quen amont et en aval de leur travail : titulaires des pousoirs de police. les militaires leur remettaient les suspeets tandis que les TPFA - interessaient prioritairement an jugement des affaires. En outre.

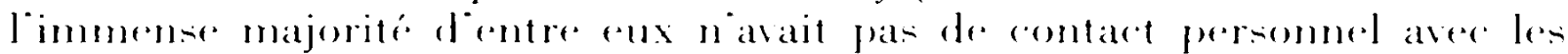
tribunaus militaires. parfois distants de plusieurs centaines de kilometres.

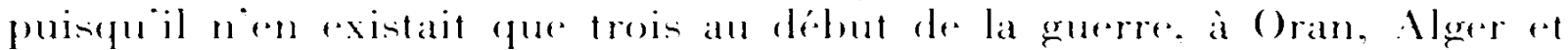
Constantine. (ies magistrats apportent ainsi des reponses teehnigues. fon-

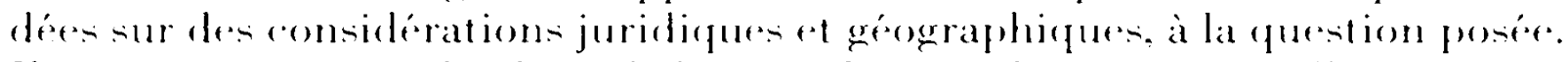
Ils se prononcent sur la phase de la provedure qui leur revenate linstruetion

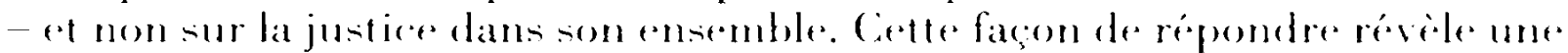
absence de consedence collective : ils sexpriment en tant que juge d"instruetion. pour la partie qui les enenerne. et pas en tant que magristrat. membre deute institution qui a ete. elle. afferter par ce transfert. Ia question mème d'une dépossesion leur semble incongrue. eomme si elle état absurde.

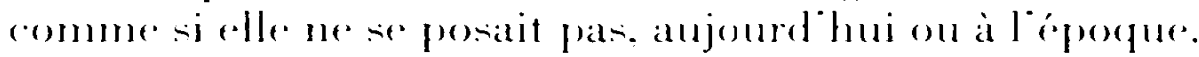

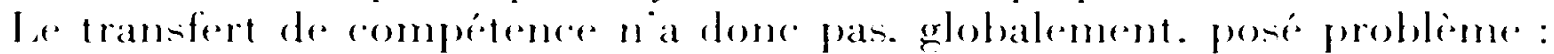

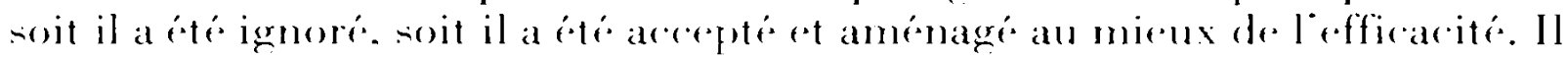
n'a plas suscite dhostilite, ni un sentiment de perte de prérogatives, de dessaisissement insoutenahle. Pourquot? I a première raison est a rechereher dans la legritimite des textes contraignant les magistrats a travailler aver la justier militaire : ladeption de ces textes a resperte le processus démocratique : légaux, ils sont incontestables. Mais anterlela de cefteraison, qui valide.

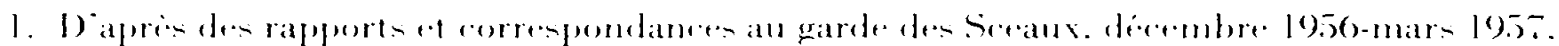

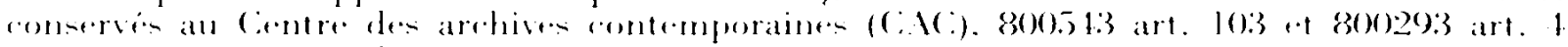

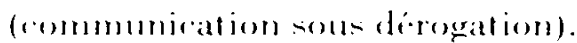

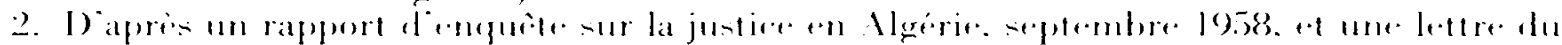

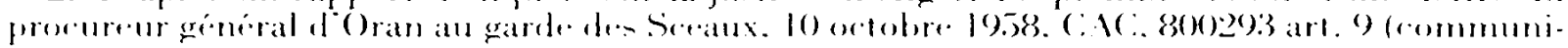
ration solus derogeation).

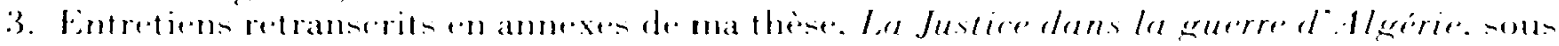

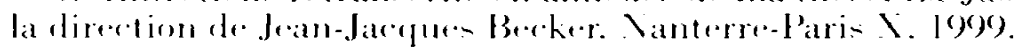

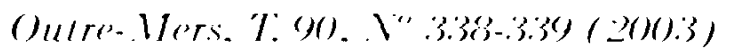


aux reux des magistrats le fait deaceepter le transfert de competence. il faut interroger le rapport au texte quentretenait la magistrature à cette éporpur.

Mlors quaujourd hui des representants re la magistrature participent a l'alaboration des textes de procédure. se prononeent sur leur reontenu. les reritiquent, protestent, il nen etait rien ente 1951 et 1962. Ponr Casamavor,

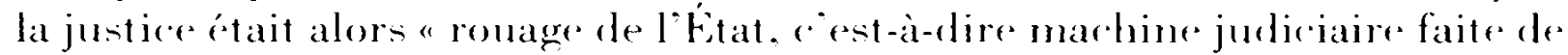
fextes ed de juges qui les appliquent... " 3. Cetle definition ferait protester les magistrats d aujourd hui qui se concoivent plus eomme les defenseurs de la

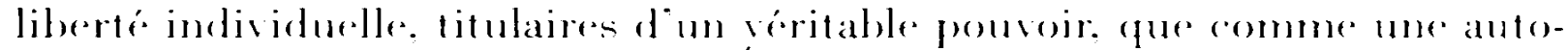
rile parmi d'autres, al serviere de létat. Lattitude des magistrats face à des

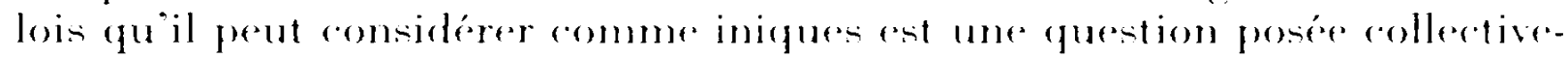
ment bien apres la guerre d' Algérie par le Syndicat de la magistrature crée.

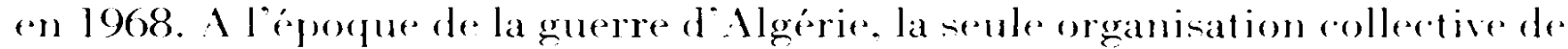
magistrats. Thion fóderale des magistrats (LWVI), etait une organisation corporatiste regroupant la quasi-totalite des magistrats de métropole, d'Outre-Mer et d'Algerie. Cette compesition lui imposat une attiturle consensuelle, mortérése alle lui interdisait toute prise de position eritique risquant de la faire aclater. Elle ne pouvait pas etre le lieu diune reflexion critique. Poser la question de lappréciation porté sur le transfert de competence vers la justice militaire à des magistrats en poste dans les annés 1950 revient ainsi a interroger cette justice avere un regard actuel. eontemporaill.

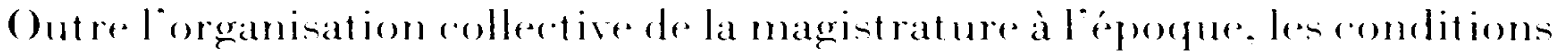
de recrutement et de formation expliquent alssis cette absenee de rapport

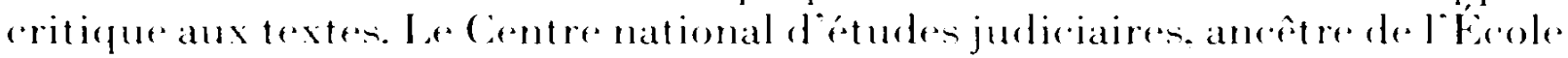
nationale de la magistrature. na ate cré qu'en 1958. I a mise en plare de cette formation professionnelle exigeant. outre une compétence juridique. une reflexion ethigue, a pris du temps. Flle a contribue, depuis. a la construetion deune irlentité collective et a la formation dou pouvoir judiciaire qui a cherehe a semanciper du pouvoir politicfue. Mais auparavant, le recrutement se faisait parmi les titulaires d une licenee en troit avant effectue un stage au barreau puis en juridietion, avant de passer un examen professionnel. Ide recrutement sur titres suivi diune formation sur le terrain. efait écalement tres pratiqué. favorisant la reproduction. de géneration en génération, des pratigues professionmelles lescales. sans recul. Dans le ras partieulier de $l^{*}$ Algerie le recrutement était deautant plus ouvert que les volontaires etaient moins nombreux. (jầce à une concurrence moins rude quen métropole, un randidat malheureux pouvait esperer intégrer la magistrature par "la petite porte algerienme". selon l'expression doun des magistrats interrogés. Ix recerutement sur titre était attractif pour des postes de juges de paix suppleants remunérés alors que les stagiaires préparant l'examen professomnel ne l'etaient pas.

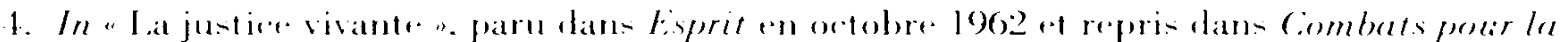
Justire. Paris. Setuil. 1968. p. 12-. 


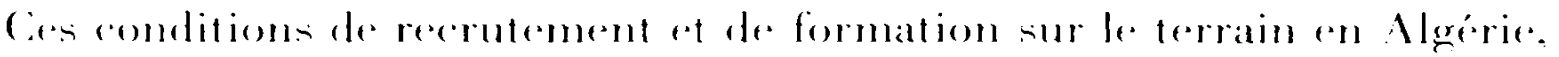

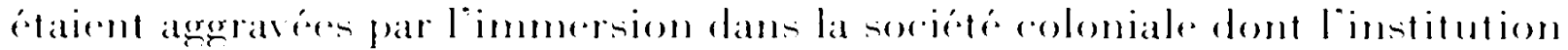

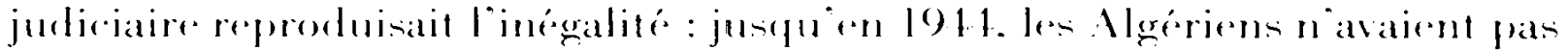
le droit dembrasser la carriere de la magist rature, seuls les postes deaxiliai-

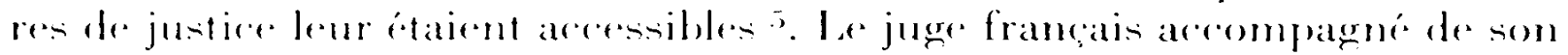

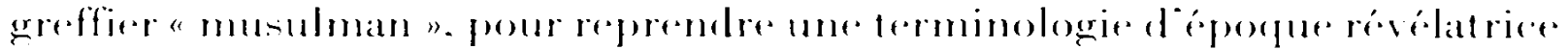

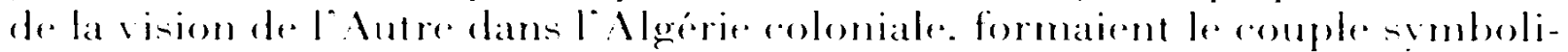

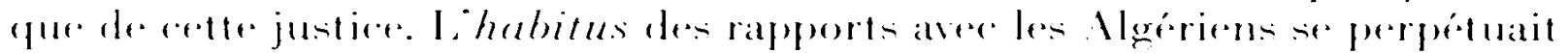
ainsi dans le cabinet du juge on an tribunal. entere des magristrats frangais.

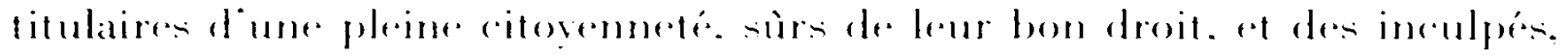

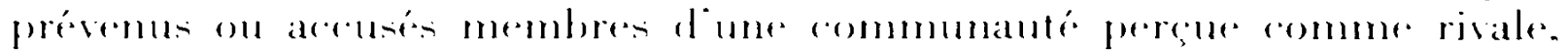

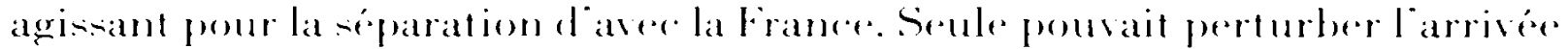
dum magistrat metropolitain portant un regard exterienr sur aes rapports

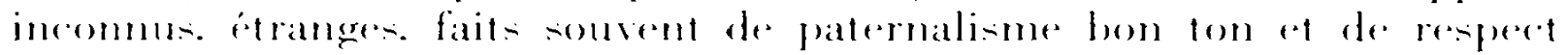
contraint. repesant parfois sur une ramaraderie on ume amitie sincere né du

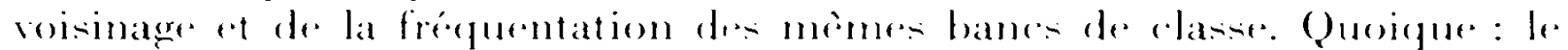

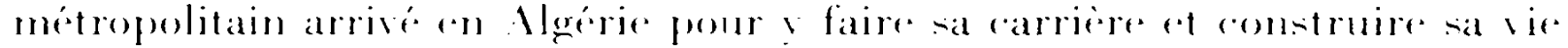

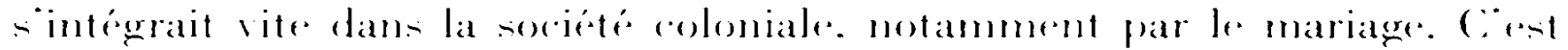

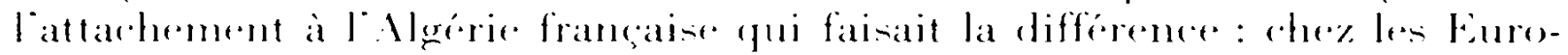

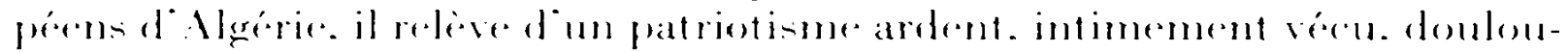

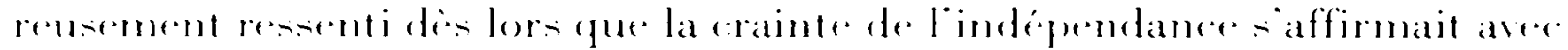

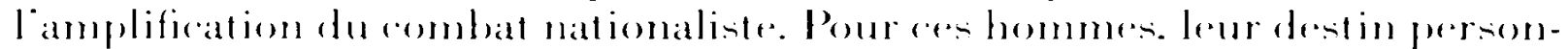
nel se jouat en mome temps que le traitement des affaters impliquant le

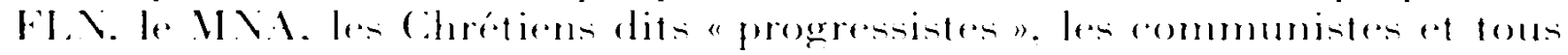
rens qui pouvaient lessoutenir.

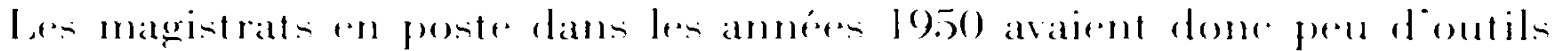

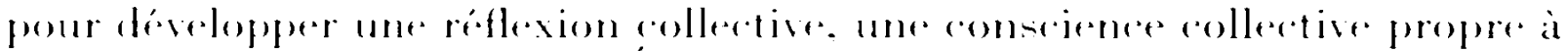

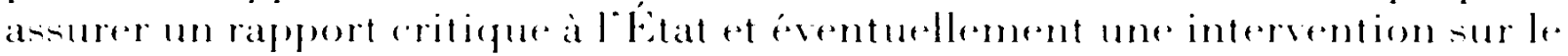

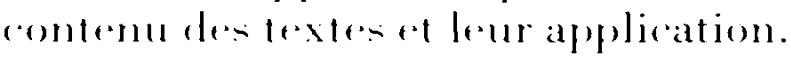

Seule la jurisprudence leur permetlat dagir. par le biais des deux plus hautes juridietions siegeant en métropole et enchappant. par eonsépuent. à

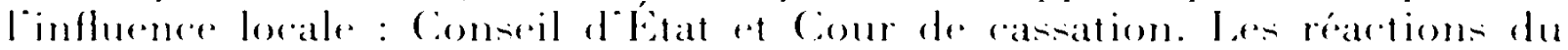

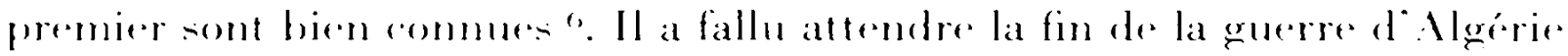
pour le voir sopposer an pouroir politique en eontestant une juridietion

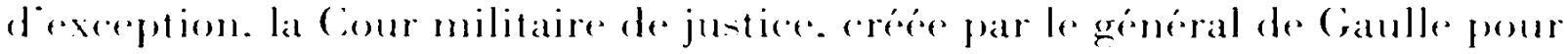

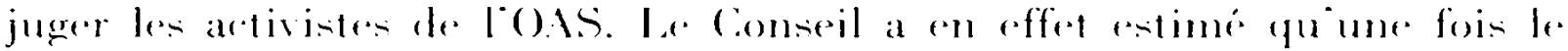

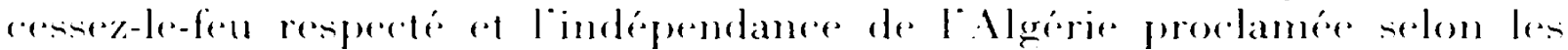
modalites prevues par les areords devian, latleinte aux principes fondamentaux du droit que portait la Cour militaire de justice naval plus lieu detre. Cet arret a tranche avere lattitude du Conseil pendant le eonflitmais. rendu le 19 octobre 1962, il appartient a lapresguerre à un moment oi les

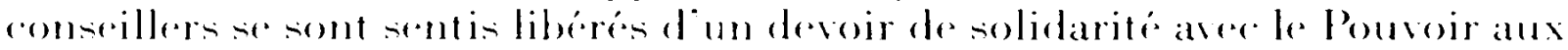

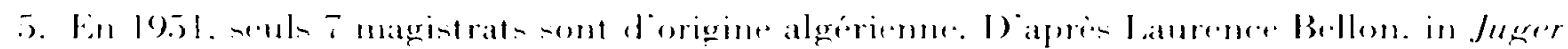

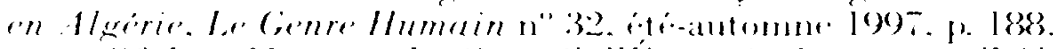

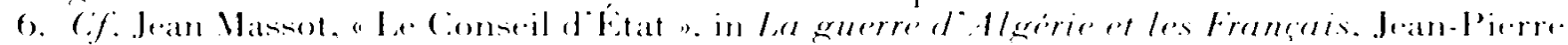

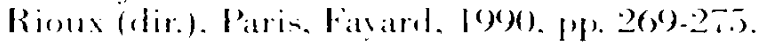

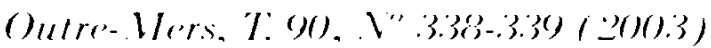


prises aner lemmemi. Pendant la guerre alueune décision semblable ne pent entremerere.

la Cour de rassation a elle aussi pris le soin de ne pas ent raver la conduite de la guerre. au point que le commandement sest folicite de ses interprétations. Pour le genéral Massu. notamment, "la jurisprudenee de la Cour de casiation est assez liberale ten ce qui concerme la definition du flagrant "rime" : d"apres lui, comme "la loi n"a pas fixé de délai de rigueur à l"expiration duquel le fait cesse d'être flagrant". "on estime généralement que par temps voisin du délit. il faut entendre une pérode de 18h" ". En dépit de la confusion du général, qui mélange crime et délit. lessent iel reste que. grâce à cette interpretation, la traduetion directe a pu etre employe dans les deux jours suivants les faits. Ce délai permettait à lamée de prendre le lemps d"interroger les prisonniers avant de les remettre a la justice. De même. la jurisprudence concernant le déeret du 12 férier 1960 créant la fonction de procureur général a pris une toumure favorable à larmée. I a diseussion portait sur le point de départ de lenquete du procureur militaire, qui disposait d'un mois pour la mener : démarraitefle le jour de l'arrestation de leindividu ou le jour de la présentation de l’individuau procureur militaire? I ans le premier cas, le procureur militaire entamait son enquete alors que l'armée renait tout juste d'arreter la personne, il etait done informe des arrestations et alait acees au prisonnier, ce qui limitait les possibilités de mauvais traitements, forture et exécutions sommaires. I ans le second ads, un lapes de temps secoulate entre larrestation re la personme et sa présentation au procureur militaire. Pendant a temps. les militaires disposaient de la persomme sans controle et le procureur militaire ne jouait plus aucun roble pour la proteretion de loindividu. Or, cest cette interprétation que la cour de cassation a rehoisi "t confirmé, dans 3 arrêts differents".

Poser la question du service de l'btat par les magristrats pendant la guerre d" Algerie revient finalement a poser la guestion duservice de lótat en lemps de guerre : pour les magistrats, il était important que leurs déeisions, leurs attitudes, ne desservent pas la guerre, nentravent pas, ne gênent pas l"armée qui la mene. D’ailleurs, quand le général Massu parle d'une jurisprudence "libérale" il veut dire une interprétation qui laisse a l"armée la liberte d"agir a sa guise. I e rapport au texte a eté surdétermine par le contexte de guerre qui a renforcé la soumission des magistrats a la volonte de ar quil est (onvenu diapeler le législateur.

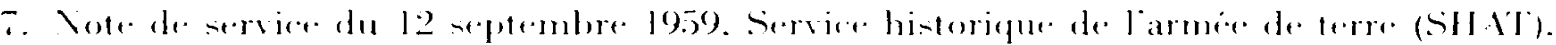
$111: 2.7(1) / 1$.

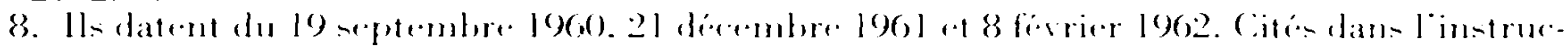

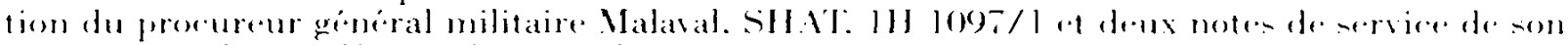

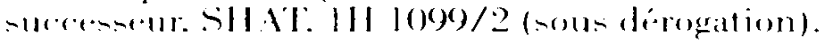




\section{Exercer des fonctions nonvelles}

La participation an TPF est le premier volet des fonctions suscitéses far la

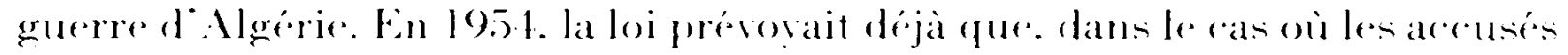
etaient des rivils. la presidenere destribunaux militaires revenait a des magis-

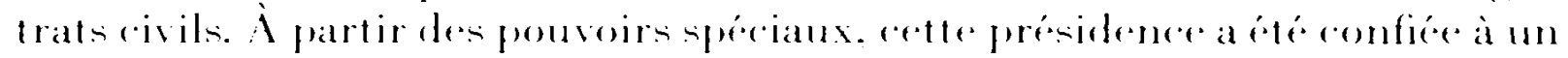

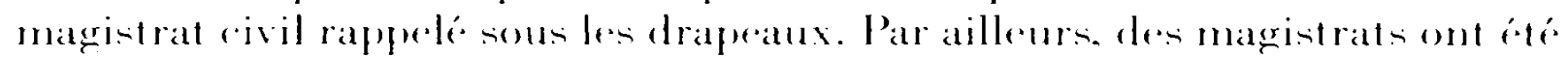

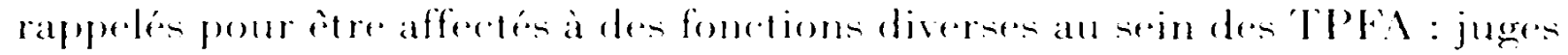
d'instruetion. substituts de juge d"instruction on substituts du commissaime

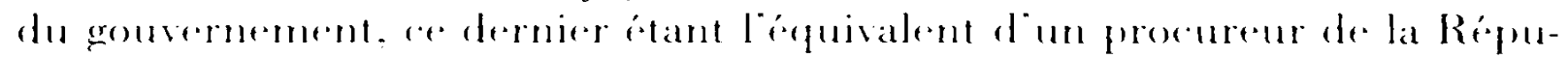
blique.

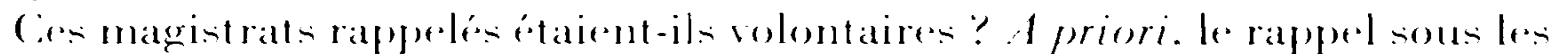

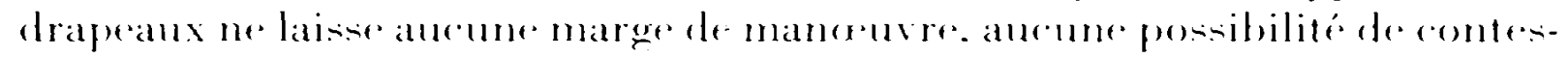

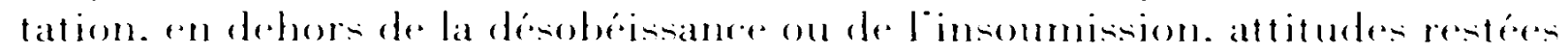
exceptionnelles. Pourtant. certains magristratsont trome la posibilite de s somstraire. Nombere dentre enx. en effete etaient radres de reserve de lat justice militaire et ils araient la posibilite den demisionmer. Ianis Bare. premier substitut an tribunal de la Seine explique ainsi que la forme du rappel laisiat toute latifute aux magistrats : "On mous enowat un flaper nous demandant si nous voulions demissomner du radre de la Justice militaire... I a magristrature militaire souffrait alors deun debut de permurie... Jai pense quil etait indigne de se deroleer... Je suis done parti pendant deux mois a (Oan puis jai préside pendant huit mois line des quatre rhambres du

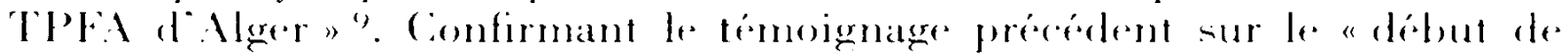

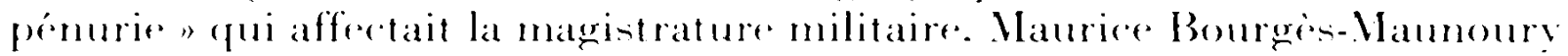

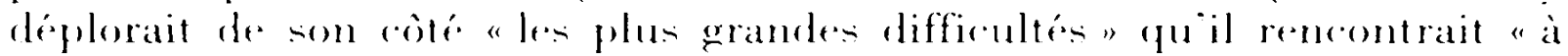
mobiliser les magestrats de loordre judiedaire de la métropoles afin de les rharger des fonctions de magistrats des tribumanx militaires en Algerie " De meme. le renforenent des struetures de la justice militaire. rendu indis-

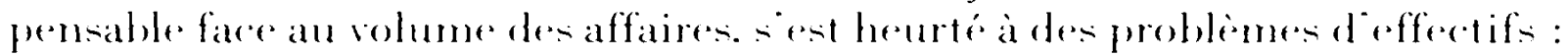
"I ees radres dactioe sont tres limites (ume rentaine de magistrats). Nos carlese de reserve sont réduits a leur plus simple expression. par suite des

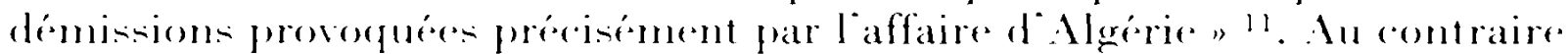
de Louis Bare. de tres nombreux magistrate ont done prefere demissionmer du radre de réserve de la justice militaire plutot que deatre rappelés et affertes dans les TPFA d' Algerie.

Cas demissions sont delicates a interpréter ar leur motivation pouvait aboir deus origines. Ite geste pouvait etre politique et manifester un refus

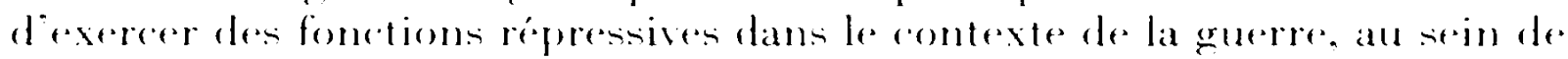

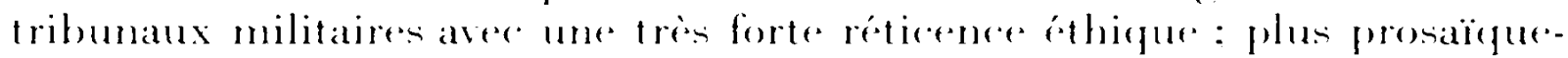

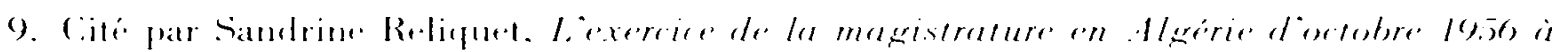

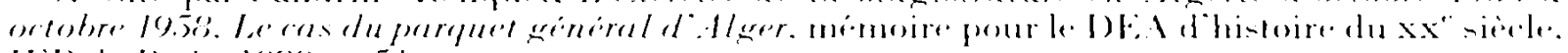
IFI' de. Pari- 1989. p. 3l.

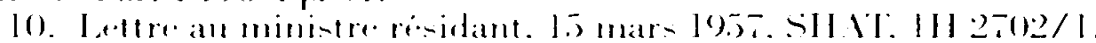

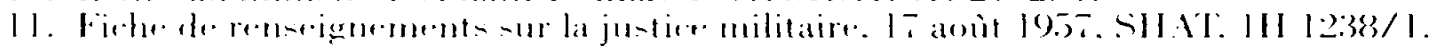

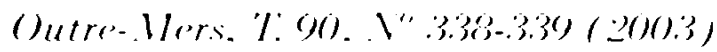


ment, les magistrats refusaient peutêtre de quitter la métropole, territoire en paix, de laisser derriere eux un travail quotidien hien rodé, d'abandonner famille et amis, pour aller aceomplir, en territoire incomnu et en guerere, une tarche nouvelle. loin des leurs. I a reaction des magistrats apparenterait alors à celle de tout rappelé, sans motivation relative à l'exereice de lewr métier; leurs réticences ne seraient pas ethiques - ou pas miquement. Ians la réalité, les deux ont puse s'entremeler dans lesprit du démissiomnaire pour former un tout farorable au refus.

A partir du déret du 12 férier 1960 , le président du tribunal militaire. ainsi que ses suppléants, ont été désgnes parmi les magistrats civils locaux, sans etre rappelés sous les drapeaux. En novembre 1960, 17 magistrats d'Algérie présidaient ains les TPHA d'Algérie dont le nombre a été porté à 13 au lien de trois ${ }^{2}$. Dans le Constantinois. par exemple, se ize magistrats, conseillers à la cour d'appel de Constantine, juges ou présidents des tribunaux de grande instance, assumaient cette tâche. Elle les oceupait beaucoup: à Constantine, le président titulaire et son premier suppléant siégeadent cing à six jours par semaine ; à Sétif. ils siégeaient chacen une semaine sur deux ; à Bone, le premier suppléant siégeait en permanenee... 1:3. Cependant. dans un aas, le magistrat sollicite sest récusé. Outre d'éventuelles réticences ethiques, les magistrats n'y trouvaient guere d'arantages : les indemnites de déplacement ne leur permettaient pas de rembourser leurs frais d'hotel si nécessaire et ils ne touchaient pas "lindemnité de risque "de $800 \mathrm{~F}$ par jour. versée aux autres membres du tribunal, alors quils étaient exposés comme eux à de possibles représailles lit. Cette situation est resté sans réponse : la seule mesure adoptée a autorisé les procureurs généraux a décharger les magristrats présidant les TPPA de leurs fonctions dans la justice civile. à rondition cependant que l'activite du TPHA le justifie et que les effectifs de la justice civile le permettent is. De facon générale. lexereice de fonctions au sein ou a la tête des TPFA a pus susciter des réserves pour des raisons tout autant politiques et ethiques que matérielles.

I a fonction de procureur militaire est lautre grande fonction exereée massivement par les magristrats en Algére pendant la guerre. Inuant les deux dernieres années de la guerre. 264 magristrats et auditeurs de justiere. rest-à-dire des jeunes magistrats sortis du Centre national d'études judiciaires. ancêtre de l'actuelle Feole nationale de la magistrature. ont ainsi été rappelés dans le cadre du déeret du 12 férier 1960 lo. Ies réticenees ne sont pas, ici. evidentes : le rappel était d'un an renowelable et certains nont pas hésité à rempiler aprés une première année d'exercice.

12. I) apres un etat des magistrats exergant en Aggerie au titre de la justice militaire, 1.5 movembre 1960. (2AC.980518 art. 2 .

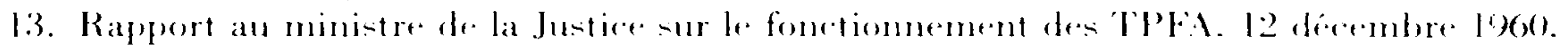
(.AC. 9.5039:5. art. 10:3.

1. Ibid.

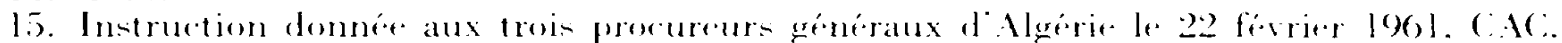
9.50 .395 .5 art 103.

16. Rapport sur lactivite des procureurs militaires redige par le procureur ginéral militaire

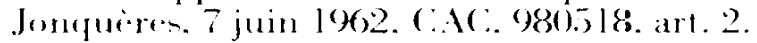




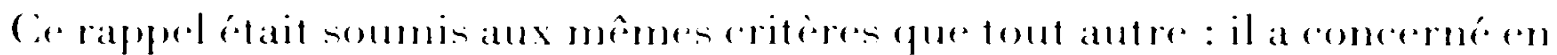

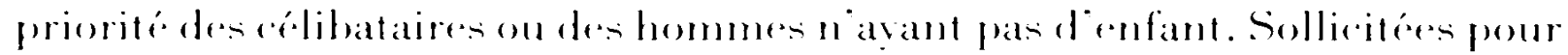

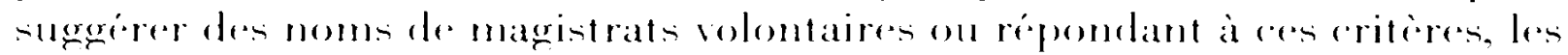

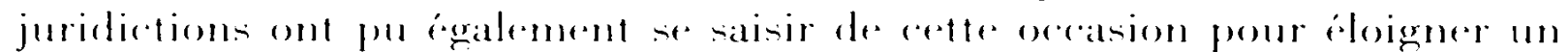

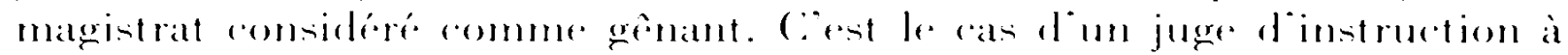

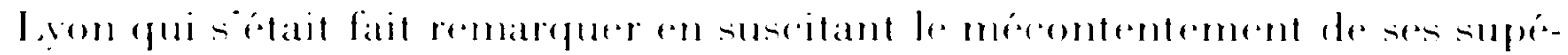

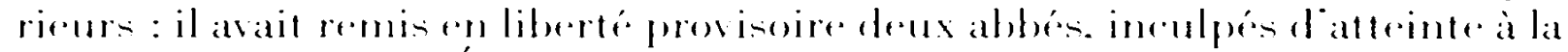

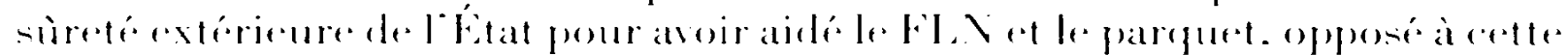

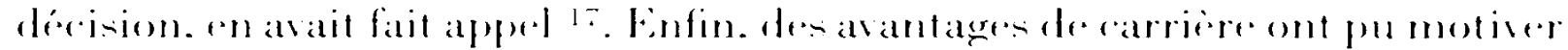

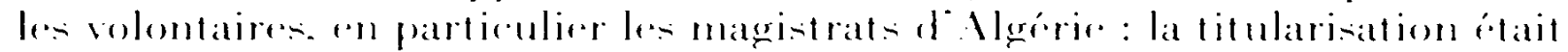
ainsi asture anx juges de paix suppleants volentaires pour un an de rappel

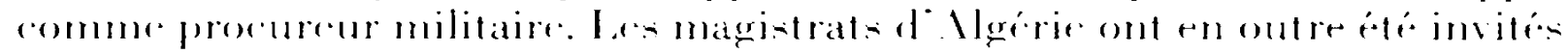
a se présenter en priorite. all molif güils commaisatent miens le terrain algerien que lears anllegues métropolitains.

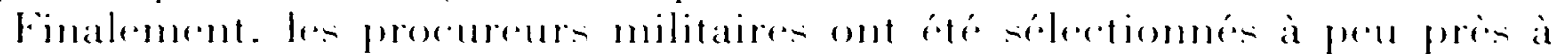

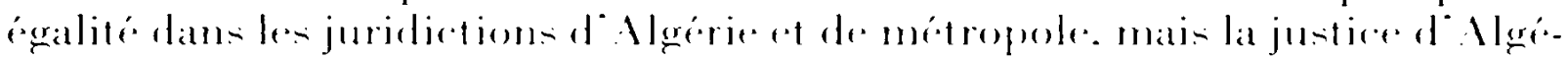

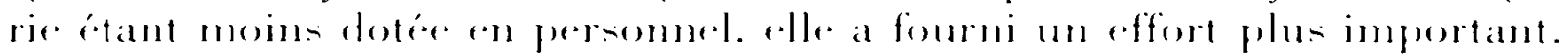

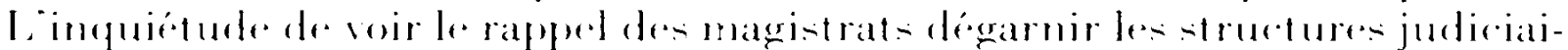

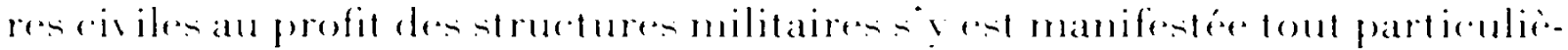

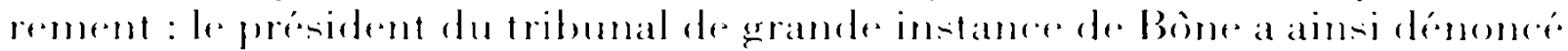

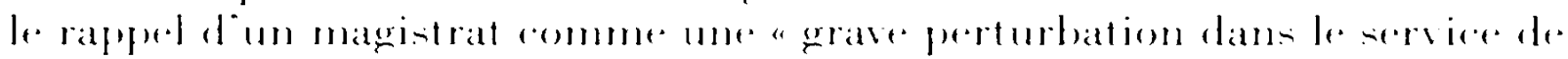

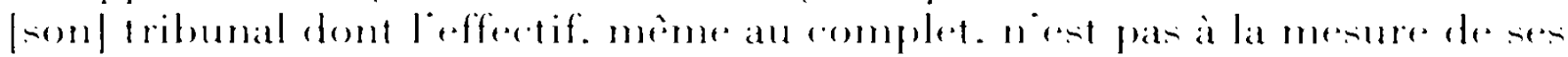

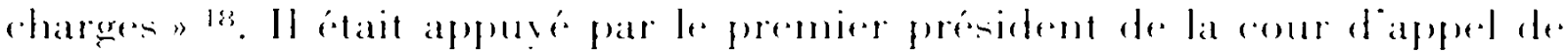

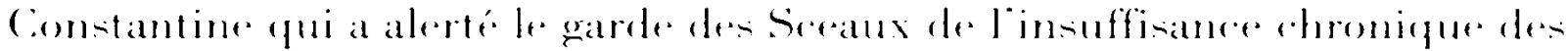

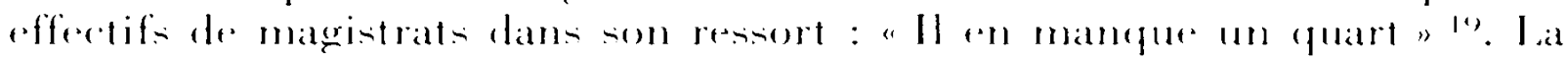

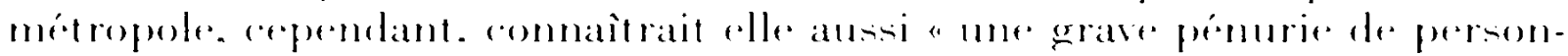

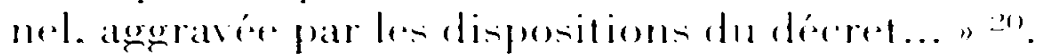

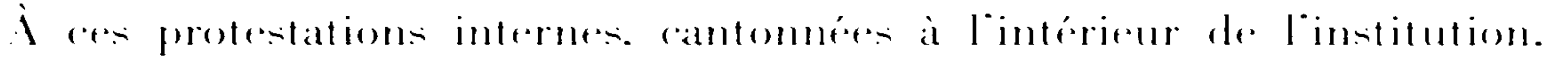

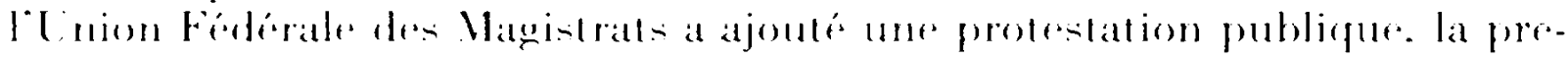

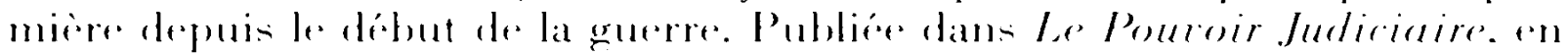

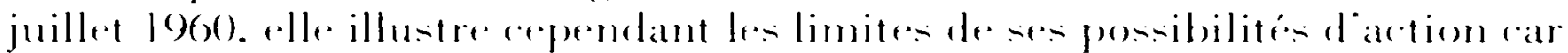

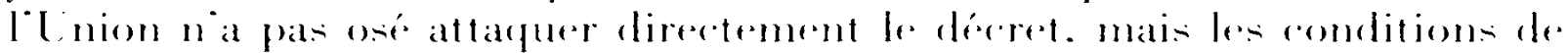
lonstallation des magistrats rappelésen Algerie : ils ant dì se tirer deaffatre aree les movens du bord en faisant appel a la comprehension des antorites militaires le plus soment. Mais eela les a placén des loabord dans la désagréa-

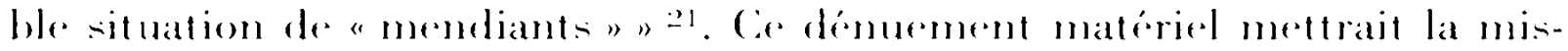
sion des procureurs militaires en péril : "..on na pas fourni aux magistrats

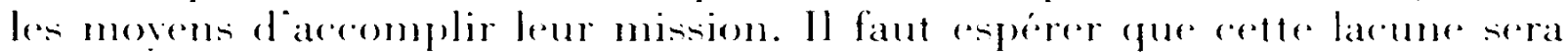

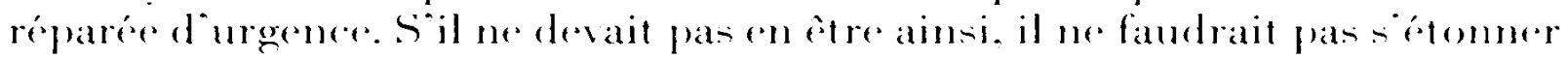

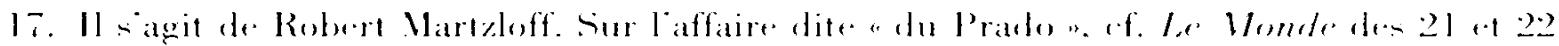
(w) 10 here 19.58.

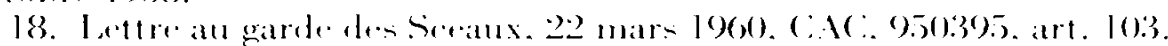

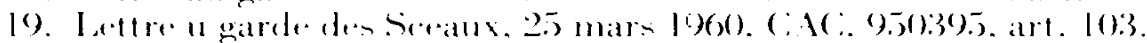

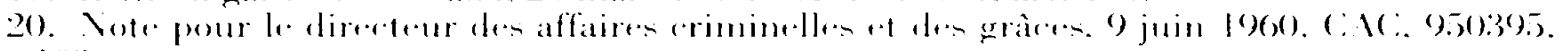
art. 10:3.

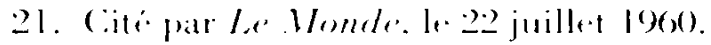

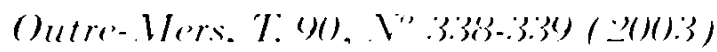


de l'echec de l'institution ". L'Cnion exprinait son scepticisme sur l'efficacité du décret du 12 février 1960 pour lutter contre les illégalités. mais elle ne pouvait pas dépasser une critique portant sur les conditions de travail des procureurs militaires : seul ce thème etait consensuel. Fille aurait risqué des désaccords et des tensions internes, si elle s'etait aventurée sur le terrain de la critique du texte en lui-meme. Ia question de son efficacité contre la pratique de la torture et des exécutions sommaires était épineuse et suscitait des avis très divergents.

Seul l'exercice de fonctions nouvelles, amplifiés ou créees dans les rirconstances de la guerre, a fait apparaître réticences et protestations. Flles témoignent d'un altachement et d'une semsibilité particuliere des magistrats à leur statut : son respect garantit l'indépendance de la Justice; des magistrats soumis a des nominations, mutations, promotions, sanctions hors d'un statut prédétermine sont effectivement a la merci du pouvoir politique. Ce postulat a très largement présidé à la fondation du Sundicat de la magistrature. Période de prise de conscience, la guerre d'Algérie a constitué dans l'histoire de la magistrature - y compris et surtout en métropole - une étape décisive dans la lente maturation conduisant, de la fin de la Seconde Guerre mondiale au début des années 1970, à la prise de distance aver le pouvoir. Ir.i. te territoire colonial en rébellion contre l'État a accentue une ciolution métropolitaine. 